\title{
Dust in Clusters of Galaxies and the Sunyaev Zel'dovich Effect $^{1}$
}

\author{
Martin Giard \\ CESR-CNRS-UPS, 9 Av. du Colonel Roche, BP 4346, F 31028 \\ Toulouse cedex 04, France
}

\begin{abstract}
We review here the unique opportunity provided by the submillimeter/millimeter domain to characterize both the warm intra-cluster plasma via the Sunyaev Zel'dovich Effect (SZE) on the cosmic microwave background, and the dust within this plasma via its thermal emission. However, multi-wavelength measurements are needed in order to separate these effects from the foreground galactic dust and the background infrared galaxies.
\end{abstract}

\section{Introduction}

Most of the mass observed in the Universe is still in the form of a diffuse gas (i.e., the "baryons") which spreads the voids between the galaxies. Within clusters of galaxies, this gas is actually a hot plasma: $T=1$ to $10 \mathrm{keV}$. The high thermal pressure is supposed to equilibrate the very deep gravitational potential imposed by the so-called "dark matter". Up to now the intra-cluster gas has been mainly studied by its thermal X-ray emission. However a new window, the millimeter/submillimeter domain, is opening for the investigation of the intracluster medium via the SZE and the thermal emission of the intra-cluster dust particles (Fig. 1). For a detailed review of the SZE, see Birkinshaw 1999 and the references therein.

\section{Dust in the Intra-cluster Medium}

\subsection{Priors}

The physics of gas-grain interactions tells us that grains similar to those that are present within galaxies should be efficiently destroyed by thermal sputtering in the hot intergalactic medium. The typical survival time for a grain of 0.1 micrometer is about $10^{8}$ years (see Dwek, Rephaeli \& Mather 1990, and Popescu et al. 2000). If we assume that all the grains from the member galaxies replenish the intergalactic medium within a time of $10^{9}$ years or more, then an upper limit of $10^{-4}$ can be put on the dust-to-gas mass ratio in the intra-cluster gas (we assume $10^{-2}$ for the dust-to-gas mass ratio within the galaxies). However,

\footnotetext{
${ }^{1}$ This work owes much to Guy Serra, PI of PRONAOS and pioneer of IR/submillimeter astronomy, who died prematurely on August 15, 2000.
} 


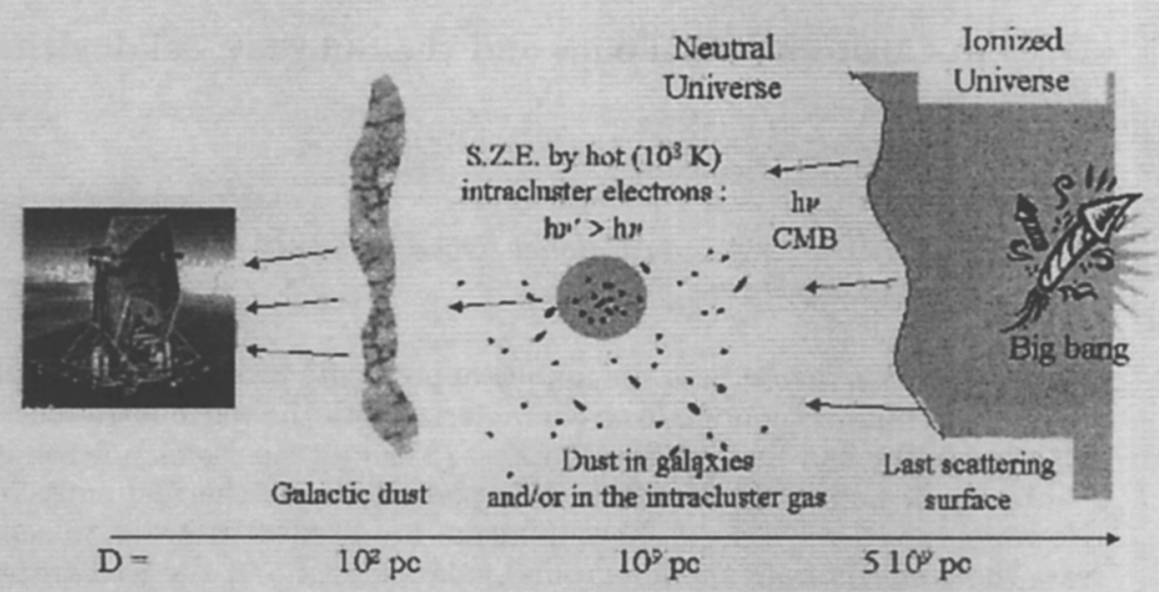

Figure 1. In the submillimeter/millimeter domain the SZ distortion of the Cosmic Microwave Background toward a cluster of galaxies is superposed on the various possible dust emissions on the line of sight: galactic dust, intra-cluster dust, and dust in the background IR galaxies.

even with this very low abundance, the total infrared luminosity of the cluster grains is comparable to its X-ray luminosity. As the grains (at $T=10$ to $20 \mathrm{~K}$ ) are heated by the hot surrounding gas, they act as a major cooling agent for the cluster baryons. There is, however, a serious observational impediment to detection of the intra-cluster IR emission: the expected surface brightness level, about $0.1 \mathrm{MJy} \mathrm{sr}^{-1}$ at $100 \mu \mathrm{m}\left(A_{v}=0.05 \mathrm{mag}\right)$, is within the fluctuation level of the foreground Galactic dust.

\subsection{Observations}

The previous physical considerations are confirmed by the direct observational fact that the $\mathrm{Fe}$ line present in cluster $\mathrm{X}$-ray spectra shows a very high gaseous $\mathrm{Fe}$ abundance : about 0.3 times the solar abundance (Fig. 2). This is very different from what happens within galaxies, where the metals are highly depleted from the gas phase, mostly hidden in grains.

Pros: Historically, Zwicky (1957) was among the first to propose the existence of dust in the general diffuse intergalactic medium (neither within the galaxies themselves, nor toward the central region of cooling flow clusters). This was to explain the lower counts of faint galaxies or distant clusters in the fields of the rich proximate clusters of galaxies. These counts required a visual extinction of the order of $A_{v}=0.3$ to $0.6 \mathrm{mag}$ (see also Karachentsev and Lipovetskii 1969). However, the direct thermal emission from this dust has been positively detected for only one cluster. This is the Coma cluster, observed with ISOPHOT at $120 \mu \mathrm{m}$ by Stickel et al. (1998). The dust emission is detected at a level of 


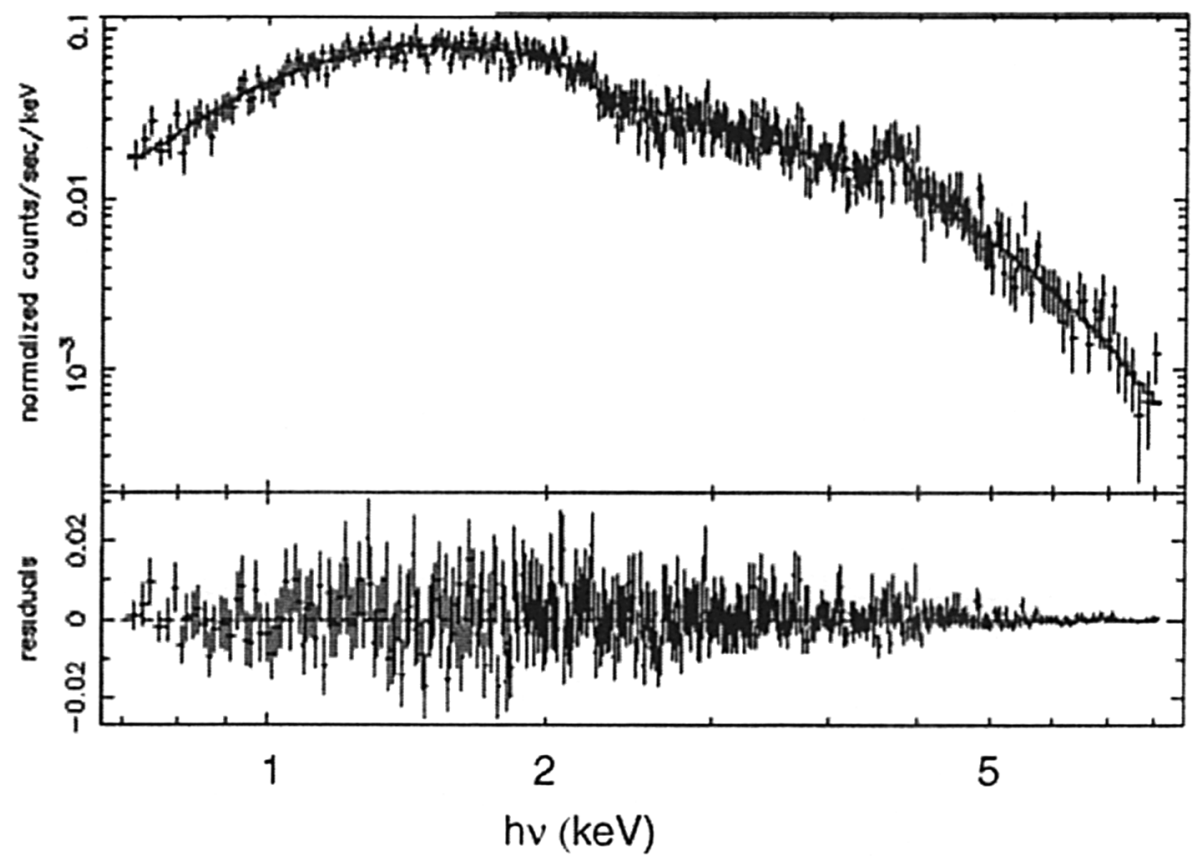

Figure 2. X-ray spectrum of the massive cluster RXJ 1347 at $z=$ 0.45 . Thermal continuum from the intra-cluster electrons at $13 \mathrm{keV}$, and emission line from gaseous $\mathrm{Fe}(\mathrm{Z}=0.33)$. This is a direct proof that $\mathrm{Fe}$ is not depleted on grains as in the Galactic interstellar medium.

about $0.1 \mathrm{MJy} \mathrm{sr}^{-1}$, corresponding to a visual extinction of $A_{v}=0.05 \mathrm{mag}$, much lower than the expectation of Zwicky. However, it is likely that faint galaxies and cluster associations are more difficult to identify within the crowded fields of rich clusters than in empty fields, resulting in the overestimation of the dust extinction by the former authors.

Cons: There are many more reports of unsuccessful searches for dust in the intra-cluster gas. From a statistical analysis of the correlation between the $\mathrm{Mg}$ absorption line and the B-V color of ellipticals in 19 nearby clusters, Ferguson (1993) derived an upper limit of $A_{v}<0.15$ (90\% confidence). Maoz (1995) obtained a similar result from the V-I colors of quasars behind rich clusters. Pointecouteau (1998) observed 5 rich clusters with ISOPHOT at 90 and $180 \mu \mathrm{m}$. They derive an upper limit of $0.15 \mathrm{MJy} \mathrm{sr}^{-1}(3 \sigma)$ for the dust emission in these clusters. Given the redshifts of these clusters, $0.1<z<0.2$, such a limit corresponds to an infrared luminosity of about $210^{10} L \odot$ (the Milky Way) for a galaxy within the cluster, or $310^{12} L \odot$ for a background object at $\mathrm{z}=1$ (equivalent to a 


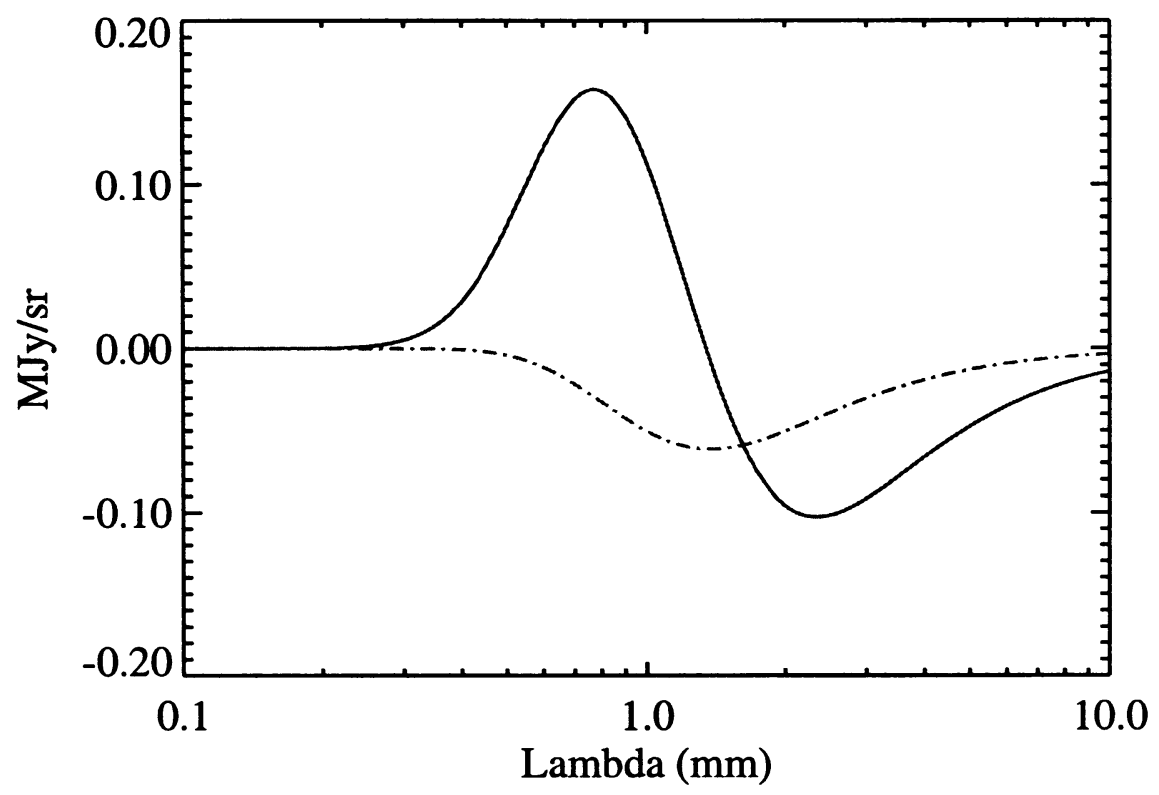

Figure 3. Spectrum of the thermal (solid line) and kinetic (dotdashed line) SZE for a Compton parameter $y=10^{-4}$, and peculiar velocity $v_{p}=3000 \mathrm{~km} / \mathrm{s}$. The velocity has been chosen very high on purpose, to clearly define the plot.

starburst like Arp 220). In terms of diffuse intra-cluster dust, the corresponding upper limit of the dust-to-gas mass ratio is $10^{-5}$.

\section{The SZ Effect}

The SZ effect is a spectral distortion of the Cosmic Microwave Background (CMB). This is produced by the hot electrons of the intra-cluster gas which efficiently scatter the CMB photons passing through the cluster. When averaging over a population of thermal electrons whose center of mass is at rest, only a small second order effect remains, with a positive bump in the submillimeter domain and a negative decrement at millimeter wavelengths : this is the socalled thermal SZE, whose amplitude relative to the CMB is directly related to the total energy of the electron cloud in the line of sight:

$$
\begin{gathered}
\frac{\Delta I_{C M B}}{I_{C M B}}=f\left(\frac{h \nu}{k T_{C M B}}\right) y \\
y=\sigma_{T} \int \frac{k T_{e}}{m_{e} c^{2}} n_{e} d s
\end{gathered}
$$


where $f$ is a dimensionless function of the photon frequency, $y$ is the so-called Compton parameter, and $T_{e}$ and $n_{e}$ are the temperature and density of the electrons.

If the whole electron cloud (i.e., the cluster) has some peculiar radial velocity component, $v_{p}$, with respect to the CMB, then this appears as a Doppler effect on the spectrum of the CMB in the direction of the cluster:

$$
\begin{array}{r}
\frac{\Delta I_{C M B}}{I_{C M B}}=a\left(\frac{h \nu}{k T_{C M B}}\right) \frac{v_{p}}{c} \tau \\
\tau=\sigma_{T} \int n_{e} d s
\end{array}
$$

where $\tau$ is the Compton optical depth.

So long as the object is resolved, the amplitude of the SZE is thus independent of the distance, contrary to all intrinsic emissions, visible, X-ray or IR, which vanish as $(1+z)^{-2}$ in a flat universe. This makes it a very powerful tool to study the ionized structure in the distant Universe. Figure 3 shows the spectrum of the thermal effect for $y=10^{-4}$. We also plot the kinetic effect of the same cluster for $v_{p}=3000 \mathrm{~km} / \mathrm{s}$. This is a high peculiar velocity, and the kinetic effect is always much smaller than the thermal effect.

Equations (1) and (2) show that the SZE itself doesn't allow us to derive the physical properties of the cluster. Complementary X-ray data are always used in a combined X-ray/SZE analysis. For instance, the electron temperature can be obtained from the high energy cut-off in the X-ray spectrum (Fig. 2). It is then possible to obtain the Compton optical depth from (1) and thus the cluster baryon mass if its distance is known. The baryon fraction is then obtained from a comparison of this baryon mass to the total mass derived from the gas temperature assuming hydrostatic equilibrium. Knowing the Compton optical depth, equation (2) then gives the radial peculiar velocity from a measurement of the kinetic effect. However, the work can be done the other way: use the optical depth derived from an X-ray analysis and estimate the gas temperature from the SZE.

Another possibility is to use the different gas density dependences of the SZE and the X-ray flux in order to estimate a typical length, $L$, of the gas cloud along the line of sight :

$$
L \propto \frac{y^{2}}{F_{X} T_{e}}
$$

Assuming spherical symmetry of the gas cloud, it is then possible to obtain the angular distance of the cluster. Together with a determination of the redshift, this gives an estimate of the Hubble constant. For distant clusters, $z>0.5$, it should be possible to obtain the deceleration parameter, $q_{0}$.

However, such determinations are heavily hampered by the fact that true clusters are neither isothermal nor spherical. Moreover, the foreground dust emission is an important pollutant. Together with the background primary CMB fluctuations, it will severely limit the measurement of the kinetic effect. In the following Section we show the first complete synthetic spectrum of the 


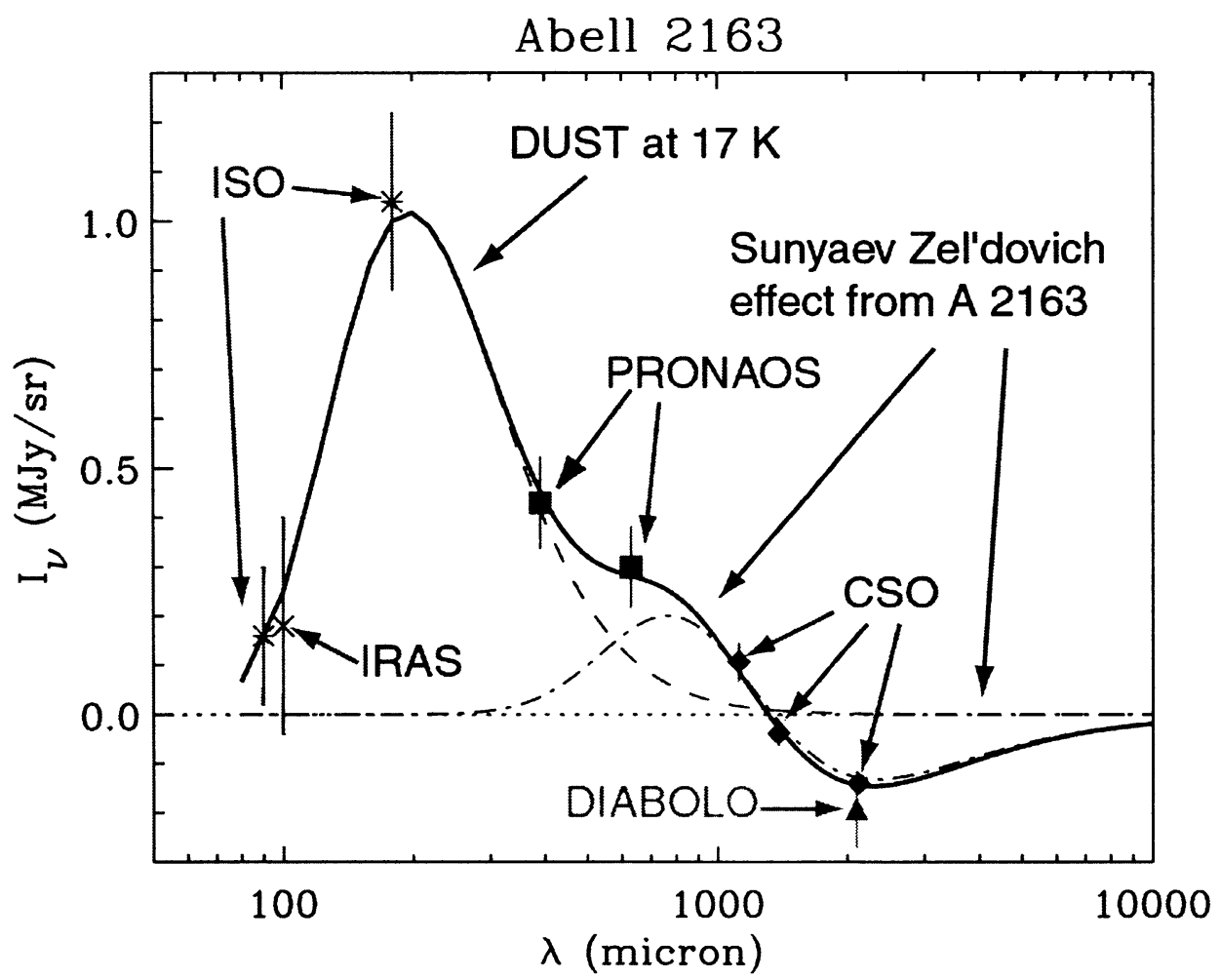

Figure 4. IR, submillimeter and millimeter measurements of the residual background toward the rich cluster of galaxies Abell 2163 . The symbols are the measurement of the excess emission (or decrement) toward the cluster relative to the sky around the cluster: beam size of 3.5 arc-min, modulation amplitude of 6 arc-min. The curve is a fit which includes thermal and kinetic SZE plus dust in our Galaxy.

submillimeter and mm background obtained in the direction of the cluster Abell 2163.

\section{Dust and SZE toward Abell 2163}

Gathering data from the PRONAOS ${ }^{1}$ balloon borne experiment and other instruments (IRAS, ISO, Suzie at CSO and Diabolo at IRAM), we were able to produce the first complete spectral measurement of the SZE toward a cluster of galaxies, Abell 2163 at $z=0.2$. PRONAOS is a 2 meter stratospheric submillimeter telescope (Fig. 6). The data presented here were obtained with two hours of integration: one hour on the cluster and equivalent time on an empty sky.

\footnotetext{
${ }^{1}$ PRONAOS is a French program funded by CNES and CNRS/INSU, see Lamarre et al. 1998
} 


\section{Submm/mm fit on A2163}

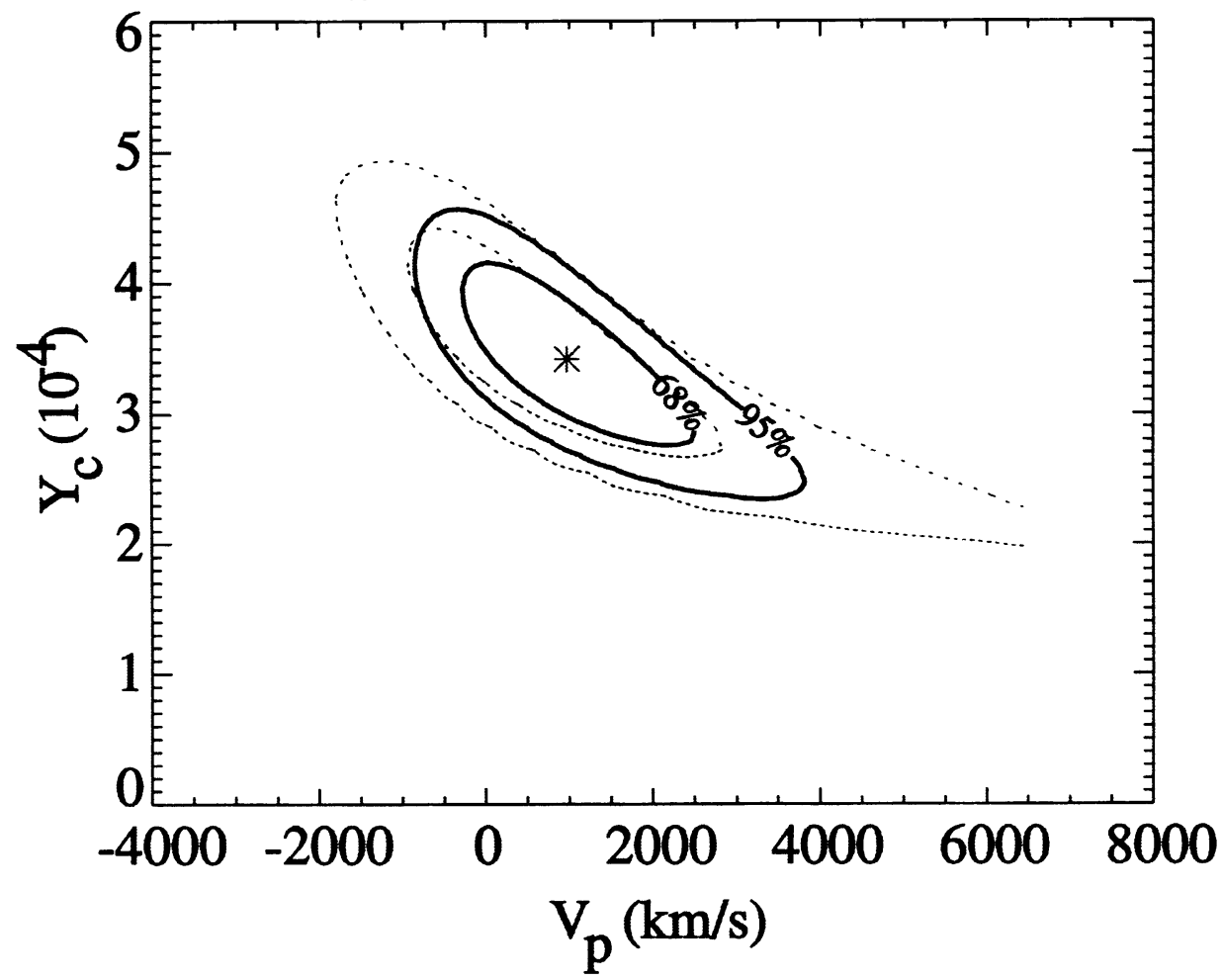

Figure 5. Derivation of the cluster Compton parameter and peculiar velocity from the IR, submillimeter and $\mathrm{mm}$ measurements toward Abell 2163: $68 \%$ and 90\% confidence levels. Dashed contours: confidence levels obtained using the $\mathrm{mm}$ ground-based measurements only.

The result is shown in Fig. 4, reproduced from Lamarre et al. (1998). The symbols show the measurement of the residual background in the direction of the rich cluster Abell 2163, relative to the background around the cluster. All measurements are normalized to the PRONAOS data: 3.5 arc-min beam and 6 arc-min modulation amplitude (which means that the reference level is taken 6 arc-min away from the cluster center on both sides). The ISO and IRAS points have been obtained by simulating the PRONAOS beam and modulation amplitude on data maps. There is clearly infrared emission pointed out by the ISO $180 \mu \mathrm{m}$ point. However, this emission does not show up on a radial profile obtained from the same data map. It is a residual gradient of the foreground Galactic emission. Abell 2163 is located at a moderate galactic latitude, behind a high level of Galactic dust emission, $28 \mathrm{MJy} \mathrm{sr}^{-1}$ at $180 \mu \mathrm{m}$, so that such a residue is not surprising. The SZ parameters from the cluster are thus recovered using a model which is the sum of dust thermal emission $(T=17 \mathrm{~K})$ and SZE 


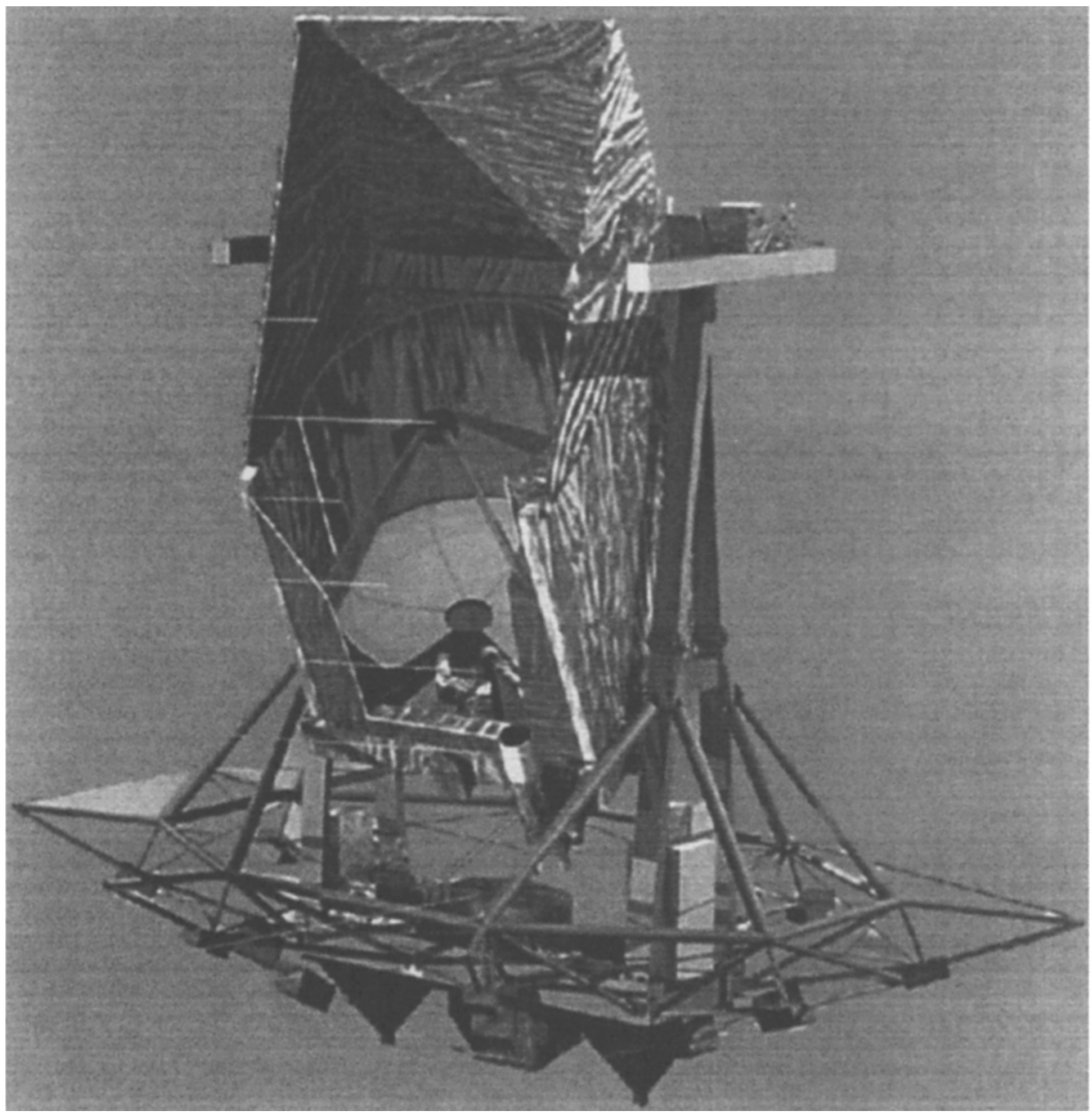

Figure 6. The PRONAOS gondola: a stratospheric 2 meter submillimeter telescope. 
(thermal and kinetic). The best model and its components are the lines in Fig. 4. The confidence levels for the $\left(y, v_{p}\right)$ parameters are plotted in Fig. 5. We also plot on the same Figure the confidence levels for a fit performed on the groundbased millimeter data only. This is to illustrate the importance of having a full spectrum. This is the only way to separate the SZE from the dust emission on the same line of sight. If this is not done, then the long wavelength tail of a dust residue (Galactic or cluster origin) will pollute the very faint kinetic SZE at $1.38 \mathrm{~mm}$.

\section{Conclusion}

Abell 2163 is a massive cluster located behind a high level of Galactic cirrus. We thus think that the parameter extraction performed on this multi-wavelength data set is representative of what will be done in the future on fainter clusters at high galactic latitudes with the much higher sensitivity data of the FIRST and Planck satellite telescopes (launch in 2007). We expect that in addition to the few thousand known Abell clusters, Planck in its all-sky survey will be able to identify a similar number of new clusters, most of them being located at high redshifts, $z>0.2$. This will certainly open a new era in the science of large scale structures.

\section{References}

Birkinshaw, M. 1999, Phys. Rept., 310, 97

Dwek, E., Rephaeli, Y., \& Mather, J. 1990, ApJ, 350, 104

Ferguson, H. 1993, MNRAS, 263, 343

Karachentsev, I. \& Lipovetskii, V. 1969, Soviet Phys., 12, 909

Lamarre, J. M., Giard, M., Pointecouteau, E., Bernard, J. P., Serra, G., Pajot, F., Désert, F. X., Ristorcelli, I., Torre, J. P., Church, S., Coron, N., Puget, J. L., \& Bock, J. J. 1998, ApJ, 507, L5

Maoz, D. 1995, ApJ, 455, L115

Pointecouteau, E. 1998, Ph.D. thesis, Université Paul Sabatier, Toulouse, France

Popescu, C., Tuffs, R., Fischera, J, Völk, H. 2000, A\&A, 354, 480

Stickel, M., Lemke, D., Mattila, K., Haikala, L. K., \& Haas, M. 1998, A\&A, 329,55

Zwicky, F. 1957, PASP, 69, 518 


\section{Discussion}

Richard Tuffs: You showed a predicted spectral energy distribution from A2163, which exhibited a peak in the $100-200 \mu \mathrm{m}$ range at a brightness of $\sim 1 \mathrm{MJy} \mathrm{sr}^{-1}$. Can you clarify the physical origin of this peak: is it a pure intracluster dust prediction, or does it include emission from constituent gas-rich galaxies?

Martin Giard: This is foreground Galactic dust. The radial profile centered on A2136 at $180 \mu \mathrm{m}$ does not show any emission to a level $\leq 0.15 \mathrm{MJy} \mathrm{sr}^{-1}$.

Jim Felten: You showed some earlier pieces of evidence about dust in clusters. Some of these suggested roughly that $A_{V} \leq 0.15$. What does your model for Abell 2163 say about $A_{V}$ ? What is your general estimate of $A_{V}$ in clusters?

Giard: 1 . The residual $1 \mathrm{MJy} \mathrm{sr}^{-1}$ detected toward A2163 is from Galactic foreground dust. 2 . $A_{V} \leq 0.05 \mathrm{mag}$, at the $90 \%$ confidence level. 